Journal for

ImmunoTherapy of Cancer

\title{
Activated but impaired IFN- $\gamma$ production of mucosal-associated invariant $T$ cells in patients with hepatocellular carcinoma
}

\author{
Wenyong Huang, ${ }^{1,2,3,4}$ Dongmei Ye, ${ }^{1,2,3}$ Wenjing $\mathrm{He},{ }^{1,2,3}$ Xiaoshun $\mathrm{He},{ }^{1,2,3}$ \\ Xiaomin Shi, ${ }^{1,2,3}$ Yifang Gao (1) ${ }^{1,2,3}$
}

To cite: Huang W, Ye D, He W, et al. Activated but impaired IFN- $\gamma$ production of mucosalassociated invariant $\mathrm{T}$ cells in patients with hepatocellular carcinoma. Journal for ImmunoTherapy of Cancer 2021;9:e003685. doi:10.1136/ jitc-2021-003685

- Additional supplemental material is published online only. To view, please visit the journal online (http://dx.doi.org/10. 1136/jitc-2021-003685).

Accepted 22 October 2021
Check for updates

(c) Author(s) (or their employer(s)) 2021. Re-use permitted under CC BY-NC. No commercial re-use. See rights and permissions. Published by BMJ.

For numbered affiliations see end of article.

\section{Correspondence to} Professor Yifang Gao; gaoyf26@sysu.edu.cn

Professor Xiaomin Shi; shixm5@sysu.edu.cn

\section{ABSTRACT}

Objective Mucosal-associated invariant T (MAIT) cells are innate T cells with immunoregulatory activity and were recently found to be associated with various tumor types. The role of intrasinusoidal MAIT cells in hepatocellular carcinoma (HCC) has not been fully characterized. Design Peripheral blood samples were obtained from patients with HCC and healthy controls. Liver-associated mononuclear cells (LMCs) were collected from liver perfusions of donors and patients with HCC undergoing liver transplantation. Blood and liver perfusates from patients with HCC were analyzed by flow cytometry for CD3 +CD161+Vo7.2+MAIT cell frequency, phenotype, and function.

Results There were fewer MAIT cells in the peripheral blood and liver of patients with HCC than in the healthy controls. Interferon- $\gamma$ (IFN- $\gamma$ ) production by these cells was also reduced. Peripheral MAIT cells showed upregulation of HLA-DR (Human Leukocyte Antigen DR ) and the inhibitory molecule PD-1 (Programmed Cell Death Protein 1), but no significant differences in upregulation were found in intrasinusoidal MAIT cells. MAIT cells were significantly enriched in the liver relative to that in the peripheral blood of patients with HCC. High levels of activation markers and exhaustion markers including HLADR, CD69, and PD-1 were observed in LMCs of patients with HCC but not in the peripheral blood. Single-cell RNA sequencing revealed that intrasinusoidal MAIT cells exhibited distinct features in patients with HCC and the controls.

Conclusion Our study showed that alterations in MAIT cells are associated with HCC. The distinct activity and function of MAIT cells in the peripheral blood and liver of patients with HCC might suggest a potential role of these cells in disease pathogenesis.

\section{INTRODUCTION}

Hepatocellular carcinoma (HCC) is the most common form of liver cancer. Although patients with HCC can be managed by surgical resection and liver transplantation, the overall 5-year survival rate remains poor. ${ }^{1}$ HCC develops slowly during chronic inflammation triggered by exposure to infectious pathogens such as hepatitis B virus (HBV) and hepatitis $\mathrm{C}$ virus (HCV), and the risk of developing HCC increases with the severity of inflammation and fibrosis. ${ }^{23} \mathrm{HBV}$ infection is a major cause of hepatitis, cirrhosis, and $\mathrm{HCC}^{4}{ }^{4}$ accounting for approximately $50 \%$ of HCC cases worldwide. ${ }^{5}$

Mucosal-associated invariant $\mathrm{T}$ (MAIT) cells in humans have innate-like characteristics and express a unique semi-invariant $\mathrm{T}$ cell receptor (TCR), including an invariant $\mathrm{V} \alpha 7.2$ and a limited array of $\mathrm{V} \beta 2$ or $\mathrm{V} \beta 13$ chains. ${ }^{6}$ In contrast to conventional $\mathrm{T}$ cells, MAIT cells are mostly CD8+, expressing high levels of CD161 on their surface. ${ }^{78}$ MAIT cells can be activated directly by MR1-presented bacterial ligands or indirectly via interleukin (IL)-12 and IL-18. ${ }^{9}$ Once activated, MAIT cells secrete proinflammatory cytokines such as interferon- $\gamma$ (IFN- $\gamma$ ) and tumor necrosis factor- $\alpha$ (TNF- $\alpha)$. A reduction in frequency and impaired function of MAIT cells was observed in the peripheral blood of patients with chronic hepatitis B. ${ }^{10}$ Most HCCs are usually associated with chronic HBV infection. It has been reported that the frequency of circulating MAIT cells is decreased in patients with colorectal cancer, ${ }^{11} \mathrm{HCC}^{12}$ and lung cancer. ${ }^{13}$ Additionally, MAIT cell frequency is diminished within liver metastases of colorectal carcinoma, and the function of hepatic tumor-infiltrating MAIT cells is impaired. ${ }^{14}$ MAIT cells are abundant in healthy adult human livers and account for approximately $20 \%-50 \%$ of all intrahepatic $\mathrm{T}$ cells. The liver serves as an immunological organ where MAIT cells are enriched and the role of MAIT cells in HCC deserves further investigation.

We aimed to analyze the phenotype and function of MAIT cells in the peripheral blood and liver of patients with HCC. Our study provides evidence that MAIT cells are 
associated with HCC and indicates a distinct function for peripheral blood MAIT cells and intrasinusoidal MAIT cells in patients with HCC. Our single-cell RNA sequencing (RNA-Seq) results showed that intrasinusoidal MAIT cells in patients with HCC belong to a population of cells with a high expression of LAG3.

\section{MATERIALS AND METHODS \\ Human samples}

Blood samples were obtained from patients with HCC $(\mathrm{n}=34)$ and age-matched controls (aged 22-73 years; $\mathrm{n}=52)$. The phenotype, immune activity, and immunosenescence of intrasinusoidal MAIT cells were assessed in patients with HCC. Liver-associated mononuclear cells (LMCs) were collected from donors $(\mathrm{n}=16)$ after portal flush, using cold $\left(0^{\circ} \mathrm{C}-4^{\circ} \mathrm{C}\right)$ preservation solution following the removal of the donor livers and from the explanted livers $(n=18)$ of patients with HCC undergoing liver surgery. The collection was performed according to standard protocols prior to liver transplantation. ${ }^{14}$ Subsequently, LMCs and peripheral blood mononuclear cells (PBMCs) were isolated using densitygradient centrifugation with Ficoll-Paque Plus (GE Healthcare, Uppsala, Sweden).

\section{Flow cytometry}

PBMCs were stained with the following antibodies: antiTCR-Va7.2 FITC (REA179, Miltenyi Biotec, GmbH), peridinin anti-CD3 PerCP-Cy5.5 (HIT3a), anti-CD4 BV510 (OKT4), anti-CD161 APC (4AHP-3G10, 4A Biotech Co. Ltd.), anti-CD8 PE-Cy7 (SK1), anti-IFN- $\gamma$ APC-Cy7 (4S. B3), anti-TNF- $\alpha$ BV421 (Mab11), anti-HLA-DR APC-Cy7 (L243), and anti-CD279 (PD-1) BV421 (EH12.2H7). All antibodies were from BioLegend, unless otherwise specified. The samples were stained at $4^{\circ} \mathrm{C}$ for $30 \mathrm{~min}$ and then processed and analyzed on a FACSCantoII machine using FACSDiva software. Flow cytometric analysis was performed using FlowJo V.10.0 for Windows.

\section{Cell sorting strategy}

MAIT cells were sorted based on CD3 +CD161+TCR Va7.2+ expression using magnetic cell separation (Miltenyi Biotec, GmbH). MAIT cells were defined as CD161 + TCR-Va7.2+CD3+ T cells (figure 1A). More than $88 \%$ purity and $90 \%$ viability were obtained after sorting by positive selection. A minimum of $4 \times 10^{4}$ cells/sample was sorted from $10^{7}$ perfusates or PBMC samples.

\section{MAIT cell activation and intracellular staining}

MAIT cells were subjected to intracellular staining for IFN- $\gamma$ and TNF- $\alpha$. Cells were stimulated with $100 \mathrm{ng} / \mathrm{mL}$ PMA (Sigma-Aldrich, USA), $1 \mu \mathrm{g} / \mathrm{mL}$ ionomycin (EMD Millipore, USA), $50 \mathrm{ng} / \mathrm{mL}$ IL-12 (PEPROTECH, USA), and $50 \mathrm{ng} / \mathrm{mL}$ IL-18 (BioLegend, USA) at $37^{\circ} \mathrm{C}$ with $5 \%$ $\mathrm{CO}_{2}$ for 6 hours prior to immunostaining. Brefeldin A $(10 \mu \mathrm{g} / \mathrm{mL})$ was added during the final 4 hours of stimulation. The immunostained samples were washed twice prior to acquisition on a FACSCantoII Flow Cytometry system (BD Biosciences).

\section{Cytokine multiplex bead-based assay and Luminex}

Cytokine concentrations in cell culture supernatants were assessed using ProcartaPlex, a multiplex bead-based immunoassay (Invitrogen), according to the manufacturer's instructions (Luminex, FLEXMAP 3D). IL-12/IL-18 and PMA (Phorbol 12-Myristate 13-Acetate) /ionomycin were used to stimulate sorted MAIT cells $(10,000$ MAIT cells per patient sample) and PBMCs/LMCs across the clinical cohorts and controls for 24 hours. The supernatants were then collected and analyzed with a cytokine human 18-plex (IL-27, TNF- $\alpha$, IL-1 $\beta$, IL-4, IL-6, IL-12p70, GM-CSF, IFN- $\gamma$, IL-13, IL-9, IL-17A, IL-10, IL-5, IL-2, IL-23, IL-18, IL-21, and IL-22) assay on the Luminex system.

\section{Single-cell RNA-Seq analysis}

The cells were sorted and combined from the perfusates of patients with HCC and healthy controls. Only cells expressing SLC4A10 and IL-18RA were designated as MAIT cells and used for further analysis based on the RNA-Seq results. Briefly, trypan blue staining was used to assess the viability of sorted cells, and the samples (cell viability $>90 \%$ ) were prepared using a $10 \times$ Genomics Single Cell 3' v2 Reagent Kit according to the manufacturer's instructions. Single-cell libraries were prepared as per the protocol and sequenced on an Illumina HiSeq $\mathrm{X}$ Ten system (Illumina). Samples were filtered for lowquality reads and unmapping sequences by importing them into CellRanger and aligned to human reference genomes (hg19, GRCh37). The unique molecular identifiers per gene were counted for each cell. Differentially expressed genes were selected based on a normalized value. Principle component analysis was performed and tSNE (t-Distributed Stochastic Neighbor Embedding) was used for dimensionality reduction. Cells were represented in two dimensions, and clusters were identified and annotated according to marker gene composition. A marker gene was defined as having an expression level $>0.25 \log$ fold higher than the mean expression value of the other subclusters and detectable expression in $>25 \%$ of the cells from the corresponding subcluster.

\section{Statistical analyses}

Differences between categorical variables were analyzed using a $\chi^{2}$ test or Fisher's exact test, whereas continuous variables were compared using the non-parametric Kruskal-Wallis test for multiple group comparisons. Correlations between two continuous variables were analyzed based on Spearman's rank correlation. Differences were considered significant at $\mathrm{p}<0.05$. GraphPad Prism V.6 software (GraphPad, La Jolla, California, USA) was used to conduct the analyses and create graphs.

\section{RESULTS}

\section{Patient cohort characteristics}

Peripheral blood samples were obtained from 34 patients with HCC and from a control cohort of 52 subjects without HCC (online supplemental table 1). Liver perfusion 
Figure 1
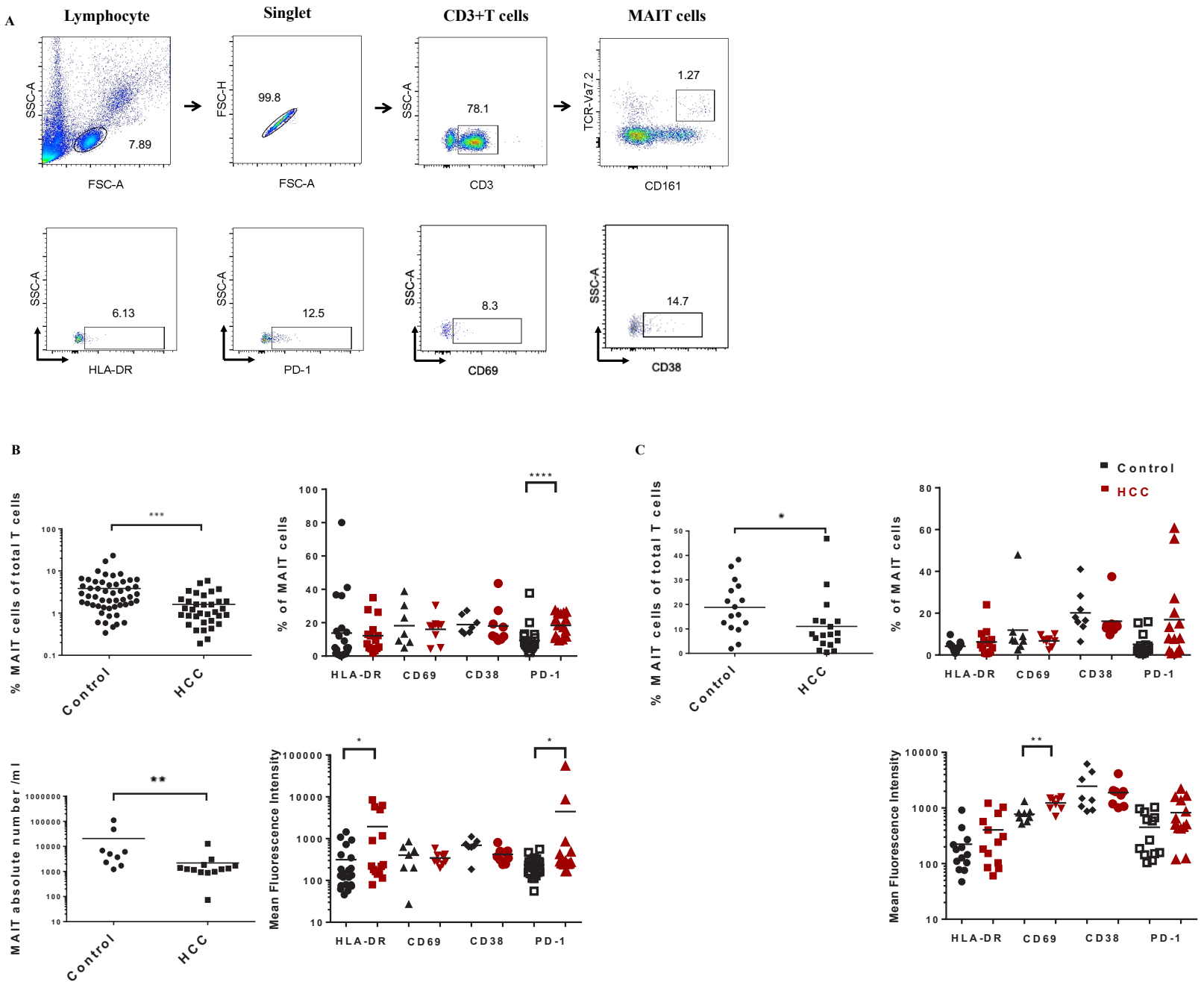

Blood

Liver

Figure 1 Mucosal-associated invariant T (MAIT) cells are severely depleted in the peripheral blood and liver of patients with hepatocellular carcinoma (HCC). (A) MAIT cells were identified using flow cytometry as lymphocytes expressing CD3, CD161, and TCR-V $\alpha 7.2$. The gating strategy of HLA-DR+, CD69+, CD38 +, and PD-1 +MAIT cells in this study is shown. (B) The frequency and absolute number of peripheral blood MAIT cells in patients with HCC $(n=34)$ and healthy control groups ( $n=52)$ were determined by flow cytometry. The percentage and expression (MFI) of HLA-DR, CD69, CD38, and PD-1 in peripheral MAIT cells were determined in patients with HCC and healthy controls. The percentage and expression levels (MFI) of HLADR, CD69, CD38, and PD-1 in intrasinusoidal MAIT cells were determined in patients with HCC (gray) and healthy donors (black). Graphs show mean and individual data points of HLA-DR (control=29, HCC=15), CD69 (control=7, HCC=9), CD38 (control=7, HCC=9), and PD-1 (control=21, HCC=15) in peripheral MAIT cells. (C) The frequency of intrasinusoidal MAIT cells in patients with $\mathrm{HCC}(\mathrm{n}=18)$ and healthy donors $(\mathrm{n}=16)$ was identified. Graphs show mean and individual data points of HLA-DR (control=13, HCC=13), CD69 (control=8, HCC=8), CD38 (control=8, HCC=8), and PD-1 (control=13, HCC=13) in peripheral MAIT cells. A Mann-Whitney $U$ test was performed to detect significant differences between groups, with asterisks indicating * $p<0.05$, ${ }^{\star \star} \mathrm{p}<0.01,{ }^{\star \star \star} \mathrm{p}<0.001$, and ${ }^{\star \star \star *} \mathrm{p}<0.0001$.

samples were collected from 18 patients with HCC and a control group of 16 donors (online supplemental table 2). All samples were collected between November 2017 and December 2019.

\section{MAIT cells are severely depleted in the peripheral blood and liver of patients with HCC}

As previously observed, ${ }^{15}$ peripheral blood and intrasinusoidal MAIT cells were significantly depleted in patients with HCC compared with levels in the controls (figure 1B,C). We then investigated the phenotype, immune activity, and immunosenescence of peripheral and intrasinusoidal MAIT cells in patients with HCC. 
Although a previous study reported that MAIT cells expressed significantly higher levels of the activating markers CD38 and HLA-DR in tumors compared with those in peritumoral or normal liver tissues, ${ }^{14}$ we observed that peripheral MAIT cells from patients with HCC express significantly higher levels of HLA-DR Mean Fluorescence Intensity(MFI) than those of controls, but there were no significant differences in the levels of CD38 in peripheral MAIT cells between patients with HCC and healthy donors. ${ }^{15}$ The percentage of cells expressing activation and exhaustion markers was not significantly different between control and HCC samples (figure 1B,C, p>0.1). However, in our study, peripheral MAIT cells of patients with HCC expressed a higher level of HLA-DR (MFI) and PD-1 (MFI) than those from the controls (figure 1B), whereas the expression of HLA-DR remained unchanged for intrasinusoidal MAIT cells. There was a tendency of higher PD-1 expression on intrasinusoidal MAIT cells from patients with HCC than those from healthy controls, although it was not significant (figure 1C). Interestingly, intrasinusoidal MAIT cells of patients with HCC expressed a higher level of CD69 (MFI) than those of controls (figure 1C). These data suggest that peripheral MAIT cells are deficient in patients with HCC.

\section{MAIT cells of patients with HCC are significantly enriched in the liver}

Our findings suggested that peripheral MAIT cells in patients with HCC expressed a higher level of HLA-DR and PD-1 than those of the controls, whereas the expression of these factors in intrasinusoidal MAIT cells remained unchanged. Then, we directly compared the frequency and activation status of MAIT cells in the peripheral blood and liver of control and patients with HCC, as well as their phenotypes and the expression levels of exhaustion and activation markers. We found that the frequency of MAIT cells was significantly higher in the liver than in the peripheral blood in both healthy control and patients with HCC (figure 2A). Moreover, HLA-DR, CD69, and PD-1 were expressed at a higher level in the peripheral blood than in intrasinusoidal MAIT cells in patients with HCC (figure 2B).

\section{Production of IFN- $\gamma$ by peripheral and intrasinusoidal MAIT cells is functionally impaired in patients with HCC}

We showed that peripheral MAIT cells of patients with HCC expressed a higher level of the immune exhaustion marker PD-1 than those of the control subjects. Although the level of PD-1 did not change significantly in intrasinusoidal MAIT cells, it showed an overall increasing trend. Since MAIT cells of patients with HCC exhibited an exhausted phenotype, we attributed this to their function being dysregulated. We first examined the changes in soluble factors in peripheral blood and the liver environment by measuring the levels of cytokines accompanying the immune response to HCC. Several key cytokines were detected by Luminex in the supernatant of PBMCs and LMCs from patients with HCC. Interestingly, the levels of cytokines such as IL-6 and GM-CSF increased in the liver of patients with HCC, compared with those in the controls, after stimulation with IL-12/IL-18 for 24 hours (figure 3A). An altered cytokine profile in the bulk culture could influence the cytokine profile of MAIT cells. We then sorted MAIT cells from PBMCs and LMCs obtained from patients with HCC and verified cytokine changes in their supernatants after stimulation with IL-12/IL-18 or PMA/ionomycin for 24 hours. IL-12/IL-18 stimulation induced a greater than fourfold increase in the levels of IL-4, IFN- $\gamma$, IL-1b, TNF-alpha, IL-13, IL-23, IL-18, and IL-21 in intrasinusoidal MAIT cells of healthy controls, compared with levels in patients with HCC (figure 3B). To gain broader insight into the cytokine profiles of MAIT cells that were affected by the bulk environment, the levels of IFN- $\gamma$ and TNF- $\alpha$ in MAIT cells of patients with HCC and controls were examined by flow cytometry. We found that peripheral blood MAIT cells from patients with HCC produced less IFN- $\gamma$ than those from the controls when IL-12 +IL-18 was stimulated (figure 3C). However, this change was not observed when cells were stimulated with PMA/ionomycin (online supplemental figure S1). These results suggested that MAIT cells from the peripheral blood were functionally impaired in patients with HCC. IFN- $\gamma$ production was also lower in MAIT cells from the liver of patients with HCC compared with control levels after stimulation with IL-12 +IL-18 (figure 3D). In agreement with the results obtained following the peripheral blood analysis, IFN- $\gamma$ production by MAIT cells did not change in response to PMA/ionomycin (online supplemental figure $\mathrm{S} 1$ ), and TNF- $\alpha$ production was not affected by any stimulation (figure 3D). These findings indicate that the function of peripheral and intrasinusoidal MAIT cells is impaired in patients with HCC.

\section{Single-cell RNA-Seq of intrasinusoidal MAIT cells of patients with HCC and healthy controls}

To better understand the characteristics distinguishing patients with HCC from the controls, single-cell analysis was performed on sorted CD3 +CD161+innate $\mathrm{T}$ cells from three pooled patients with HCC and two controls. Single-cell RNA-Seq analysis revealed 854 differential genes (with 169 upregulated and 685 downregulated in HCC) between patients with HCC and healthy controls, suggesting the presence of an aberrant gene expression profile in MAIT cells of patients with HCC. In total, 15,472 and $6241 \mathrm{CD} 3+\mathrm{CD} 161+\mathrm{T}$ cells were sequenced in the samples from controls and patients with HCC, respectively. All cells from the patients and controls expressed KLRB1 as expected. The percentage of cells expressing SLC4A10 was approximately $7 \%$ in both samples, with $12 \%$ of cells in the control samples expressing ABCB1 and only $7 \%$ in the HCC patient samples. We further filtered the MAIT cells as suggested in a previous publication, ${ }^{15}$ and post-filtering with SLC4A10/IL-18RA, 236 cells remained in the control sample $(1.5 \%)$ and 141 cells remained in HCC samples (2.3\%). An overview of threedimensional and two-dimensional t-SNE maps of MAIT 
A

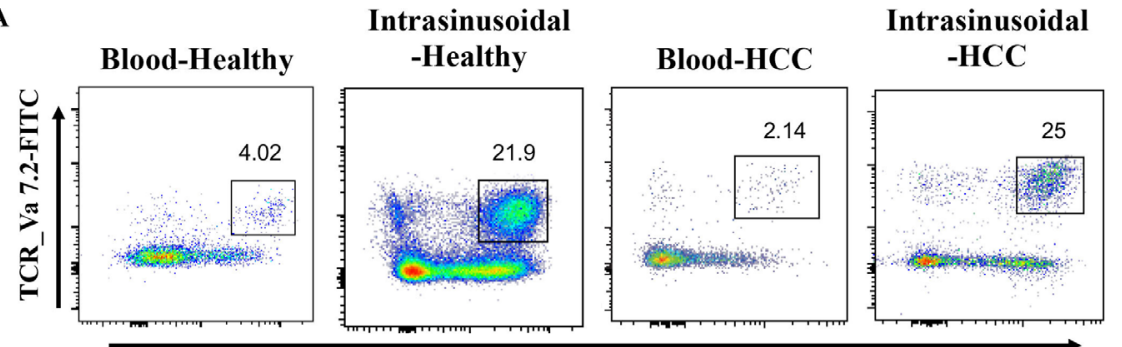

CD161-APC

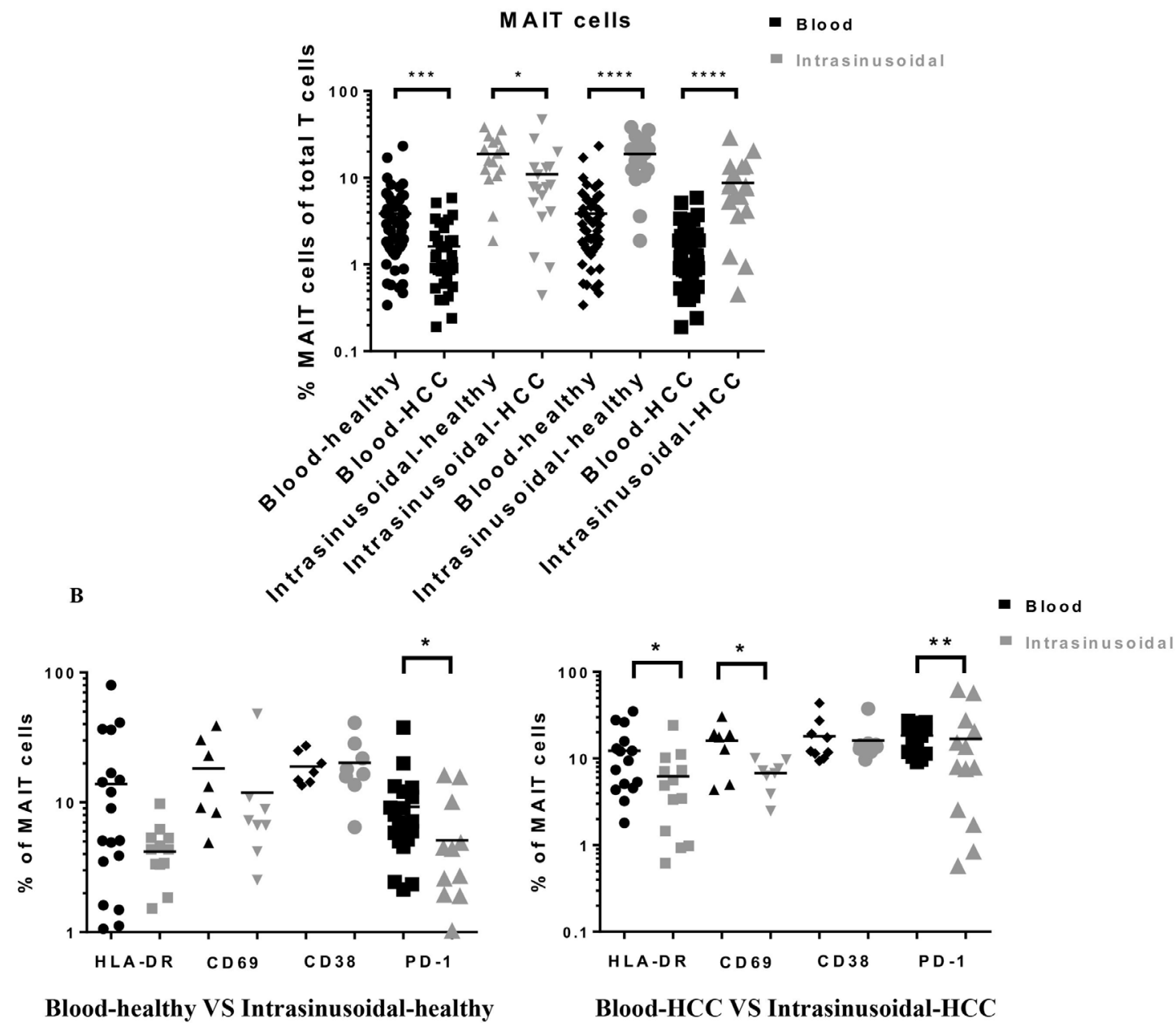

Figure 2 Expression of immune activation markers and cytokines in peripheral and intrasinusoidal mucosal-associated invariant T (MAIT) cells of patients with hepatocellular carcinoma (HCC). (A) Comparison of the proportion of MAIT cells in the blood (control=52, HCC=34) and liver (control=18, HCC=16) of patients with HCC and healthy controls. (B) Comparison of the percentage of cells expressing HLA-DR, CD69, CD38, and PD-1 in peripheral (black) and liver (gray) MAIT cells of patients with HCC. (A, B) Graphs show the mean and individual data points. A Mann-Whitney $U$ test was performed to detect significant differences between groups, with asterisks indicating ${ }^{*} \mathrm{p}<0.05,{ }^{\star *} \mathrm{p}<0.01,{ }^{\star \star \star} \mathrm{p}<0.001$, and ${ }^{\star \star \star \star} \mathrm{p}<0.0001$.

cell clusters from HCC and control samples is shown in figure 4A. Marker genes with considerable differences in expression between patients and controls are represented as a heatmap and listed in figure 4B. The exhaustion profile and gene activation were further analyzed in the patient and control samples (figure 4C). Interestingly, a marked increase in the level of LAG3 was also detected. LAG3 is known as a key T cell checkpoint and inhibitor of $\mathrm{T}$ cell responses. Together, these results suggested that the MAIT cells present in the HCC samples are activated but have an exhausted phenotype. The pathways in which the relevant key molecules are implicated are shown in figure $4 \mathrm{D}$.

\section{Intrasinusoidal MAIT cells positively correlated with plasma levels of ALT and AST}

Finally, we verified whether there was any clinical correlation between MAIT cells and the clinical status of the patients. In patients with HCC, a strong correlation was found between the frequency of intrasinusoidal MAIT 


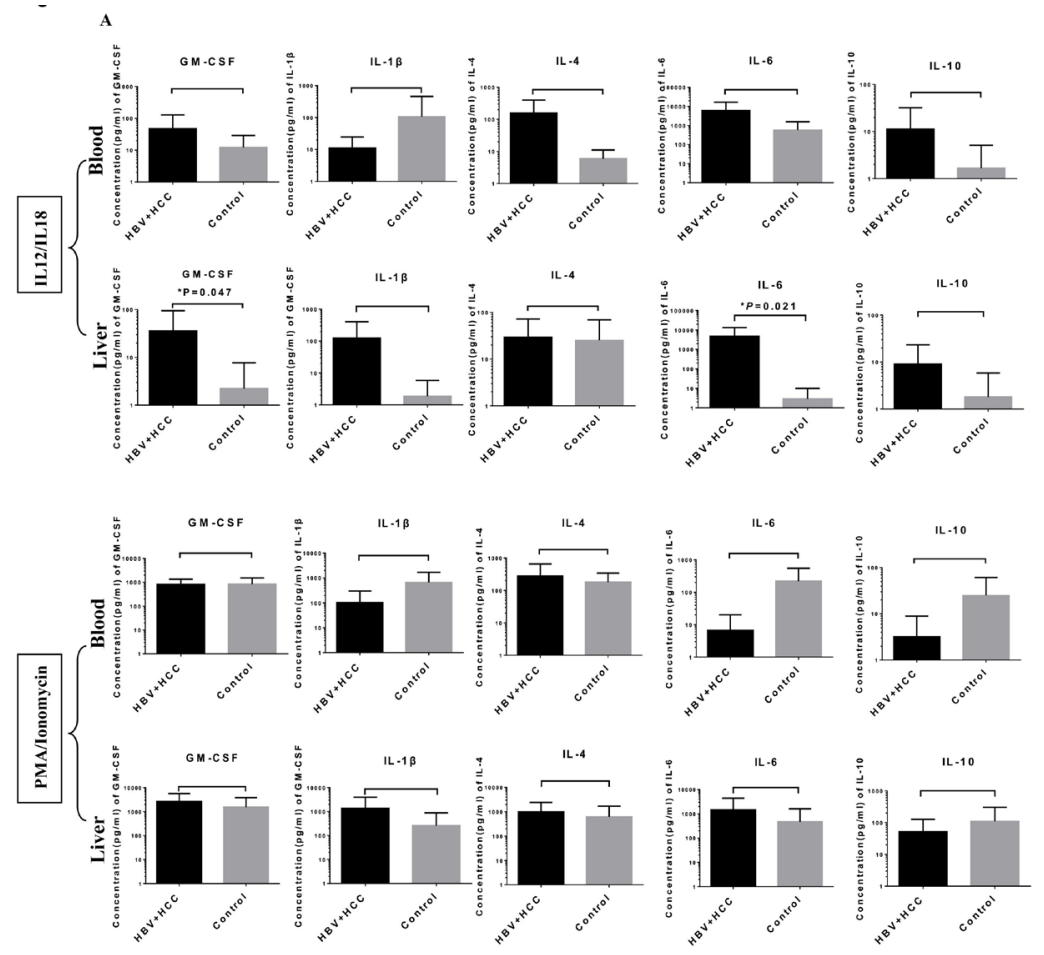

B

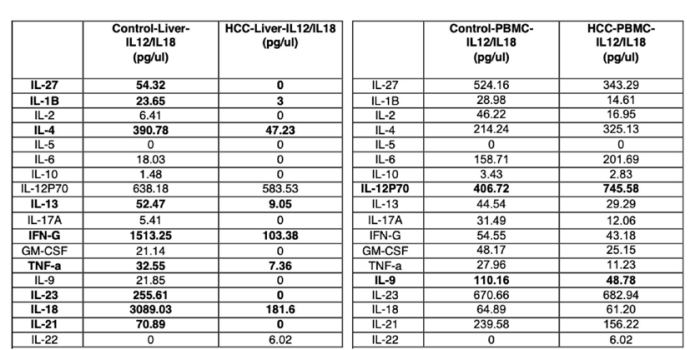

C
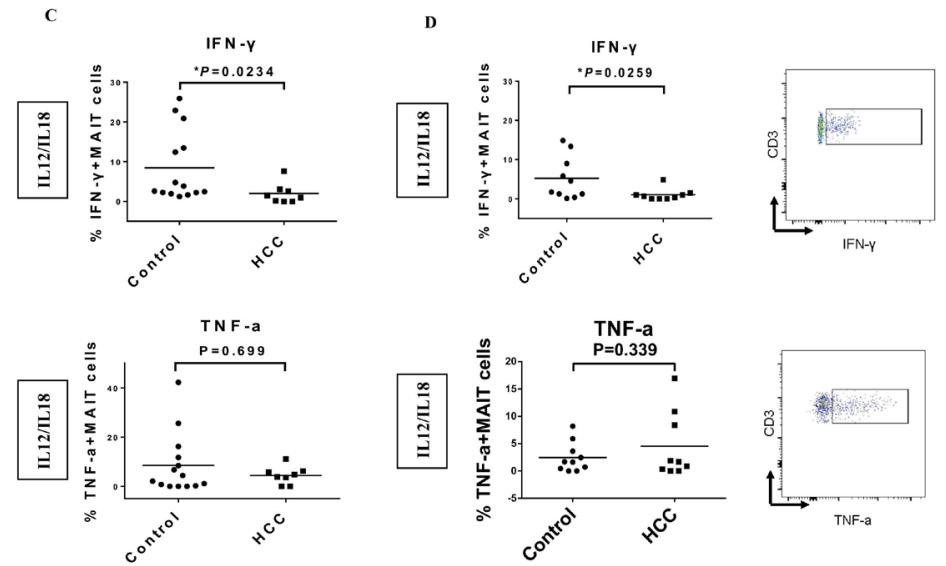

Blood

Liver

Figure 3 Cytokine expression in peripheral blood and intrasinusoidal mucosal-associated invariant T (MAIT) cells in patients with hepatocellular carcinoma (HCC). (A) Expression of cytokines in the supernatant of blood and liver cells in patients with HCC after stimulation with IL-12/IL-18 for 24 hours (control=5, HCC=8). (B) Expression levels of cytokines in the supernatant of sorted MAIT cells from the blood and liver of control $(n=3)$ and patients with HCC $(n=3)$ after stimulation with IL-12/IL-18 for 24 hours. (C) Comparison of the percentages of IFN- $\gamma$ and TNF- $\alpha$ in MAIT cells from the peripheral blood of patients with HCC $(n=8)$ and healthy controls $(n=14)$ after stimulation with IL-12/IL-18 for 6 hours. (D) Comparison of the percentages of IFN- $\gamma$ and TNF- $\alpha$ in intrasinusoidal MAIT cells of patients with HCC $(n=9)$ and healthy donors $(n=10)$ after stimulation with IL-12/IL-18 for 6 hours. (C, D) Graphs show mean and data points. The percentage of IFN- $\gamma+$ and TNF- $\alpha+$ MAIT cells after stimulation with IL-12/ IL-18 was calculated after subtracting the blank control. A Mann-Whitney $U$ test was performed to detect significant differences between groups, with asterisks indicating ${ }^{*} \mathrm{p}<0.05$, ${ }^{* \star} \mathrm{p}<0.01,{ }^{* \star *} \mathrm{p}<0.001$, and ${ }^{* * \star *} \mathrm{p}<0.0001$. HBV, hepatitis $\mathrm{B}$ virus; HCV, hepatitis $C$ virus; IFN- $\gamma$, interferon- $\gamma$; IL, interleukin; TNF- $\alpha$, tumor necrosis factor- $\alpha$. 
Figure 5
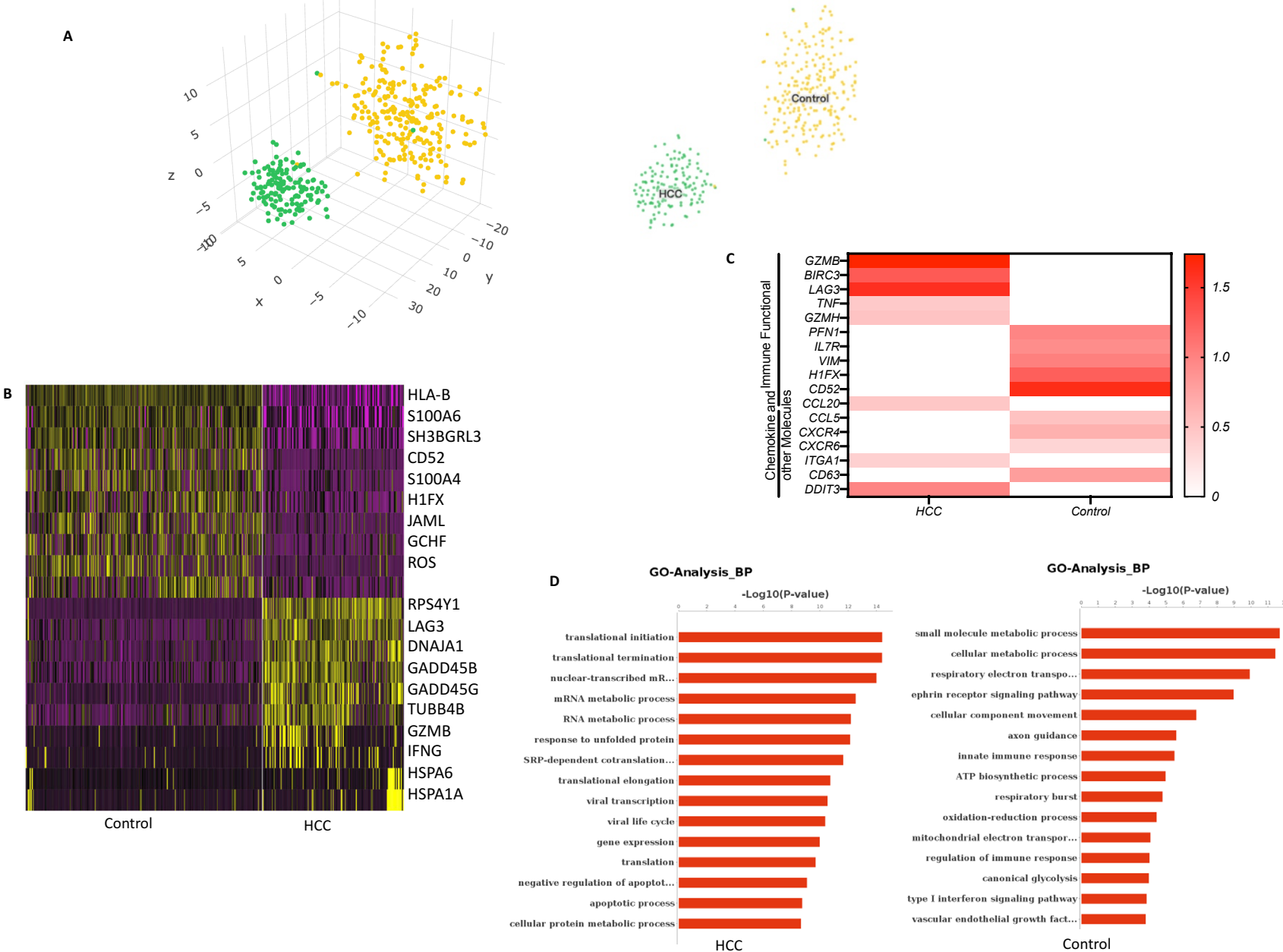

Figure 4 Comparison of gene expression patterns between patients with hepatocellular carcinoma (HCC) and healthy control subjects. (A) The cells were filtered according to SLC4A10 and IL18RA expression and the three-dimensional plot representations of tSNE analysis of mucosal-associated invariant T (MAIT) cells from controls (236 cells) and patients (141 cells). (B) Marker genes with the top differences in expression between patients and controls are presented by a heatmap. (C) The heatmap of the two-way hierarchical clustering of immune marker genes (Immune function and chemokine/cytokine profiles) from patients with HCC and controls shows distinct expression patterns of activation and exhaustion. (D) Pathways of highly expressed genes in HCC and controls. HBV, hepatitis B virus; HCV, hepatitis C virus; IFN- $\gamma$, interferon- $\gamma$; IL, interleukin; TNF- $\alpha$, tumor necrosis factor- $\alpha$.

cells and the levels of ALT (alamine aminotransferase) and AST (Aspartate aminotransferase) (figure 5B). Interestingly, this correlation was not observed in peripheral MAIT cells (figure 5A). This result suggested that intrasinusoidal MAIT cells might serve an important role in the regulation of liver function.

\section{DISCUSSION}

Alterations in innate $\mathrm{T}$ cells are associated with various cancers. ${ }^{11-17}$ MAIT cells are a population of innate T cells considerably enriched in the liver, ${ }^{18}$ which suggests their potential role in HCC. In keeping with a previous study, ${ }^{12}$ we found that the frequency of MAIT cells was reduced both in the peripheral blood and in the liver of patients with HCC. Our results further demonstrated that MAIT cells from patients with HCC exhibited an activating and exhausted phenotype with impaired effector capability. In addition, the percentage of perfusate MAIT cells was significantly correlated with ALT and AST levels in patients with HCC, which might be indicative of a poor clinical outcome. Our findings also provided novel insights into the role of peripheral blood and intrasinusoidal MAIT cells during HCC progression. In contrast to MAIT cells in colorectalcancer ( CRC), MAIT cells mainly expressed CD8 and had inflammatory functions in HCC, whereas MAIT cells comprised a subset expressing CD4 and Foxp3, suggesting a regulatory function present in the CRC environment.

The decreased frequency of circulating MAIT cells has been reported in chronic infections, such as HBV and $\mathrm{HCV}^{19}{ }^{20}$ as well as in colorectal ${ }^{11}$ and lung cancer. ${ }^{13}$ Our results show that MAIT cells are decreased in the peripheral 
A
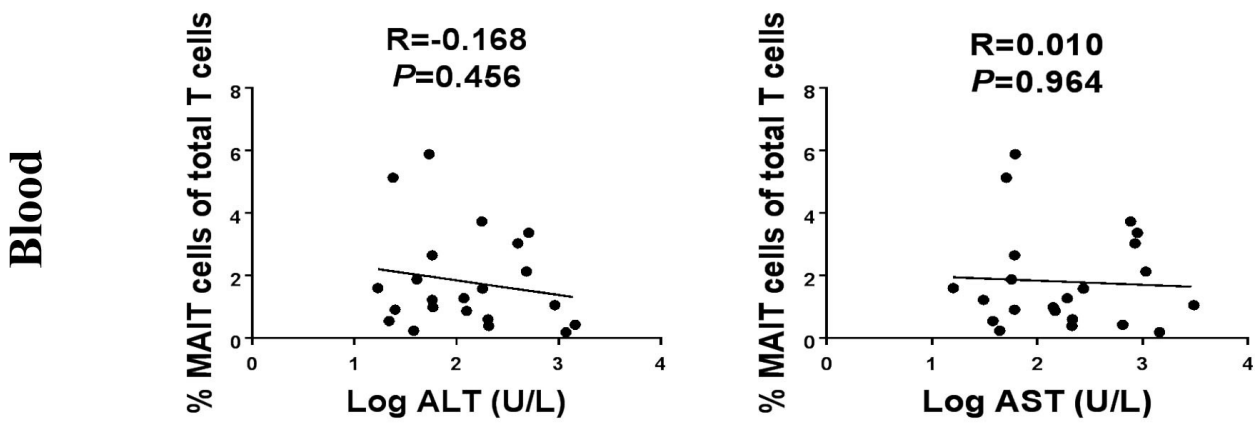

B
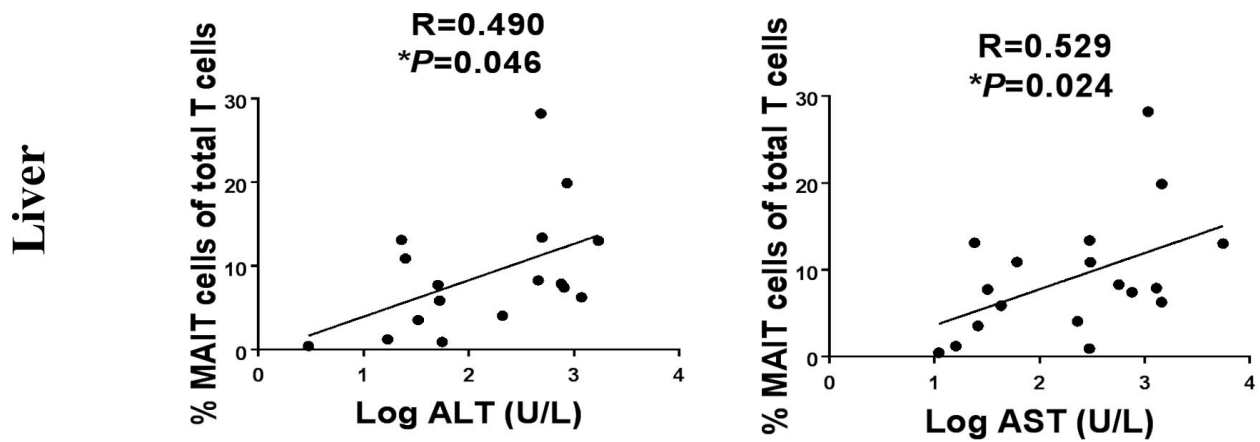

Figure 5 Correlation between mucosal-associated invariant T (MAIT) cells and the plasma levels of ALT or AST. The correlations between the plasma levels of ALT (Alamine aminotransferase) or AST (Aspartate aminotransferase) and the frequency of peripheral $(A)$ or liver $(B)$ MAIT cells in patients with hepatocellular carcinoma $(\mathrm{HCC})$ were analyzed using Spearman's test.

blood of patients with HCC. As HCC usually develops in the background of chronic viral infections, whether the reduction is attributable to tumor-associated factor or chronic infection conditions remains controversial. Results from our limited number of non-HBV-HCC patients showed the same MAIT cell pattern as HBV-HCC patients, suggesting the reduction might be caused by the tumor environment (data not shown). Additionally, the MAIT cells in peripheral blood highly express HLA-DR and PD-1, which could be explained by depletion by apoptosis subsequent to sustained activation..$^{21}$ Independent of MR1 binding, MAIT cells can produce effector cytokines such as IFN- $\gamma$ and TNF and promote antiviral immune responses. ${ }^{22}{ }^{23}$ However, in patients with HCC, the effector function of intrasinusoidal MAIT cells was found to be severely impaired. We found that IFN- $\gamma$ production was lower in HCC-sourced MAIT cells than in the controls after stimulation with IL-12/IL-18. Additionally, whereas most cytokines were not detected in sorted intrasinusoidal MAIT cells from patients with HCC, a wide range of effector cytokines was found after 24 hours of stimulation in control MAIT cells. It is possible that MAIT cells are reprogrammed within the HCC microenvironment, diminishing effector cytokine production. To characterize the cytokines secreted from the liver and in the peripheral blood of patients with HCC, we examined the cytokines present in the supernatant of PBMCs and LMCs of patients with HCC. Higher production of IL-6 and GM-CSF was observed in the supernatant of LMCs after stimulation with IL-12/IL-18 for 24 hours. The IL-6/STAT3 pathway has been reported to play a crucial role in the prevention of $\mathrm{HCC},{ }^{24}{ }^{25}$ and the levels of cytokines such as IL-6 and GM-CSF are elevated in patients with HCC. ${ }^{12} 2627$ A previous study suggested that the elevation in GM-CSF production might contribute to MAIT cell loss. Thus, it is possible that suppressing effector cytokine production by HCC resulted in intrasinusoidal cells. ${ }^{28}$ IL-12 and IL-18 can also stimulate the monocytes in PBMCs and LMCs of patients with HCC and produce several kinds of cytokines, which might skew the cytokine profile of MAIT cells. To better characterize the cytokines produced by MAIT cells in patients with HCC, these cells were sorted from the PBMCs and LMCs. The results further suggested that in the presence of an exhausted phenotype, MAIT cells display a very limited effector function.

To further investigate the immune activation status of MAIT cells in the liver and peripheral blood, we measured activation and exhaustion markers such as HLA-DR, CD69, CD38, and PD-1 in these cells. The levels of HLA-DR and PD-1 were higher in the peripheral blood of patients with HCC than 
in the controls. This might indicate that in HCC, MAIT cells are present with an activating exhausted phenotype, which has been related to tumor promotion. In addition, high PD-1 expression in MAIT cells could suggest their impaired function in patients with HCC, which would agree with our flow cytometry results that IFN- $\gamma$ expression level in MAIT cells of patients with HCC is lower than in the controls. Interestingly, our single-cell analysis found that the genes involved in type I interferon signaling were expressed in control MAIT cells. Our single-cell analysis of intrasinusoidal MAIT cells from patients with HCC further demonstrated that genes required for activation and exhaustion were highly expressed in the HCC samples. Together, these results may suggest that the effector function and cytotoxic activity of MAIT cells from patients with HCC were deficient. Duan et al previously suggested that that the reduced infiltration of MAIT cells in HCC is unlikely to be related to apoptosis. ${ }^{12}$ However, our single-cell analysis suggested that genes involved in apoptotic pathways were found to be expressed in HCC-MAIT cells.

Single-cell RNA-Seq analysis provided new insights into the function of intrasinusoidal MAIT cells in patients with HCC, based on the expression of marker genes. Significant differences in functional immune markers were observed between patients with HCC and the controls, including activationassociated and exhaustion-associated genes. Most strikingly, the exhaustion marker LAG3 was found to be highly expressed in patient samples compared with levels in the controls. LAG3 is known as a key molecule involved in T cell activation, and a recent study by Wang et alsuggested that the inhibitory function of LAG3 can be independent of Major Histocompatibility complex M (MHC) class II, ${ }^{29}$ which raised the question of whether the LAG3 present in MAIT cells could function without interacting with MHC-II. Here, we first demonstrated that LAG3 might potentially cause MAIT cell dysfunction in HCC. Previously conducted RNA-Seq determined the global gene expression differences by sorting MAIT cells from paired tumor and peritumor liver tissues, as well as normal liver of healthy donors. ${ }^{12}$ Their results showed that genes aberrantly upregulated in tumor-infiltrating MAIT cells included HAVCR2 (TIM-3). TIM-3 is also known as a checkpoint receptor expressed by a wide variety of immune cells. ${ }^{30}$ Combined with their findings, we speculate that the dysfunction of liver MAIT cells in HCC may be due to abnormally elevated immune checkpoints in MAIT cells.

Finally, patients with a high proportion of intrasinusoidal MAIT cells exhibited a three-log elevation in the levels of ALT and AST, compared with those in patients with fewer intrasinusoidal MAIT cells. This suggests that MAIT cells, particularly intrasinusoidal MAIT cells, may be predictors of liver function in patients with HCC. Previous study in HCC results showed that patients with high MAIT cell infiltration had significantly poorer RFS (Regional Free Survial) and relatively lower OS compared with the low infiltration group. ${ }^{12}$ We believe that the persistent presence in the liver might be associated with continuous inflammation in the liver in patients with HCC. Our findings suggest that activated MAIT cells could be associated with the severity of the intrasinusoidal liver environment in patients with HCC.
MAIT cells are increasingly known as immune protectors, particularly during infectious diseases. Here, we demonstrated that intrasinusoidal MAIT cells in the tumor environment of HCC might not favor antitumor responses owing to the presence of an exhausted phenotype and diminished effector responses. Therefore, MAIT cell reprogramming in the tumor setting might be an attractive therapeutic strategy for HCC.

\section{Author affiliations}

${ }^{1}$ Organ Transplantation Center, The First Affiliated Hospital, Sun Yat-sen University, Guangzhou, People's Republic of China

${ }^{2}$ Guangdong Provincial Key Laboratory of Organ Donation and Transplant Immunology, The First Affiliated Hospital, Sun Yat-sen University, Guangzhou, People's Republic of China

${ }^{3}$ Guangdong Provincial International Cooperation Base of Science and Technology (Organ Transplantation), The First Affiliated Hospital, Sun Yat-sen University, Guangzhou, People's Republic of China

${ }^{4}$ Department of Pathology, The Second Affiliated Hospital of Nanchang University, Nanchang, People's Republic of China

Contributors WH, DY, and WH: Acquisition of data. WH and DY: Analysis and interpretation of data. YG, XS, XH, and WH: Study concept and design. XS and YG: Study supervision, drafting of manuscript, and obtained funding. YG: Acting as guarantor for overall content.

Funding YG is supported by the National Natural Science Foundation of China (Grant numbers: 31800758,32070882$)$. SX is supported by the National Natural Science Foundation of China (Grant numbers: 31800753), Guangdong Basic and Applied Basic Research Foundation (Grant numbers: 2019A1515010639) The organ transplantation center is supported by Guangdong Provincial Key Laboratory of Organ Donation and Transplant Immunology, The First Affiliated Hospital, Sun Yat-sen University, Guangzhou, China (2013A061401007, 2017B030314018) and Guangdong Provincial International Cooperation Base of Science and Technology (Organ Transplantation), The First Affiliated Hospital, Sun Yat-sen University, Guangzhou, China (2015B050501002).

Competing interests None declared.

Patient consent for publication Not applicable.

Ethics approval This study was conducted in accordance with the ethical principles stated in the Declaration of Helsinki. This study was fully approved by the First Affiliated Hospital of Sun Yat-sen University ethical board (2018)073. All participants provided written informed consent before sample collection.

Provenance and peer review Not commissioned; externally peer reviewed.

Data availability statement All data are available and can be shared upon request.

Supplemental material This content has been supplied by the author(s). It has not been vetted by BMJ Publishing Group Limited (BMJ) and may not have been peer-reviewed. Any opinions or recommendations discussed are solely those of the author(s) and are not endorsed by BMJ. BMJ disclaims all liability and responsibility arising from any reliance placed on the content. Where the content includes any translated material, BMJ does not warrant the accuracy and reliability of the translations (including but not limited to local regulations, clinical guidelines, terminology, drug names and drug dosages), and is not responsible for any error and/or omissions arising from translation and adaptation or otherwise.

Open access This is an open access article distributed in accordance with the Creative Commons Attribution Non Commercial (CC BY-NC 4.0) license, which permits others to distribute, remix, adapt, build upon this work non-commercially, and license their derivative works on different terms, provided the original work is properly cited, appropriate credit is given, any changes made indicated, and the use is non-commercial. See http://creativecommons.org/licenses/by-nc/4.0/.

\section{ORCID iD}

Yifang Gao http://orcid.org/0000-0003-2817-9391 


\section{REFERENCES}

1 Li J, Huang L, Yan J, et al. Liver resection for hepatocellular carcinoma: personal experiences in a series of 1330 consecutive cases in China. ANZ J Surg 2018;88:E713-7.

2 Parikh P, Ryan JD, Tsochatzis EA. Fibrosis assessment in patients with chronic hepatitis B virus (HBV) infection. Ann Trans/ Med 2017;5:40.

3 Carmona I, Cordero P, Ampuero J, et al. Role of assessing liver fibrosis in management of chronic hepatitis $\mathrm{C}$ virus infection. Clin Microbiol Infect 2016;22:839-45.

4 Mast EE, Alter MJ. Epidemiology of viral hepatitis: an overview. Semin Virol 1993;4:273-83.

5 El-Serag HB. Epidemiology of viral hepatitis and hepatocellular carcinoma. Gastroenterology 2012;142:1264-73.

6 Treiner E, Duban L, Bahram S, et al. Selection of evolutionarily conserved mucosal-associated invariant T cells by MR1. Nature 2003;422:164-9.

7 Walker LJ, Kang Y-H, Smith MO, et al. Human MAIT and CD8 $\alpha \alpha$ cells develop from a pool of type-17 precommitted CD8+ T cells. Blood 2012;119:422-33.

8 Le Bourhis L, Martin E, Péguillet I, et al. Antimicrobial activity of mucosal-associated invariant T cells. Nat Immunol 2010;11:701-8.

9 Jo J, Tan AT, Ussher JE, et al. Toll-like receptor 8 agonist and bacteria trigger potent activation of innate immune cells in human liver. PLoS Pathog 2014;10:e1004210.

10 Yong YK, Tan HY, Saeidi A, et al. Decrease of CD69 levels on TCR $\mathrm{V} \alpha 7.2^{+} \mathrm{CD} 4^{+}$innate-like lymphocytes is associated with impaired cytotoxic functions in chronic hepatitis $B$ virus-infected patients. Innate Immun 2017;23:459-67.

11 Ling L, Lin Y, Zheng W, et al. Circulating and tumor-infiltrating mucosal associated invariant T (MAIT) cells in colorectal cancer patients. Sci Rep 2016;6:20358.

12 Duan M, Goswami S, Shi J-Y, et al. Activated and exhausted MAIT cells foster disease progression and indicate poor outcome in hepatocellular carcinoma. Clin Cancer Res 2019;25:3304-16.

13 Won EJ, Ju JK, Cho Y-N, et al. Clinical relevance of circulating mucosal-associated invariant $\mathrm{T}$ cell levels and their anti-cancer activity in patients with mucosal-associated cancer. Oncotarget 2016;7:76274-90.

14 Shaler CR, Tun-Abraham ME, Skaro Al, et al. Mucosa-associated invariant $\mathrm{T}$ cells infiltrate hepatic metastases in patients with colorectal carcinoma but are rendered dysfunctional within and adjacent to tumor microenvironment. Cancer Immunol Immunother 2017;66:1563-75.

15 Zheng C, Zheng L, Yoo J-K, et al. Landscape of infiltrating T cells in liver cancer revealed by single-cell sequencing. Cell 2017;169:1342-56.

16 Sundström P, Ahlmanner F, Akéus P, et al. Human mucosaassociated invariant $T$ cells accumulate in colon adenocarcinomas but produce reduced amounts of IFN- $\gamma$. J Immunol 2015;195:3472-81.

17 Peterfalvi A, Gomori E, Magyarlaki T, et al. Invariant Va7.2-Ja33 TCR is expressed in human kidney and brain tumors indicating infiltration by mucosal-associated invariant T (MAIT) cells. Int Immunol 2008;20:1517-25.

18 Jo J, Tan AT, Ussher JE, et al. Toll-like receptor 8 agonist and bacteria trigger potent activation of innate immune cells in human liver. PLoS Pathog 2014;10:e1004210.

19 Huang W, He W, Shi X, et al. Mucosal-associated invariant T-cells are severely reduced and exhausted in humans with chronic HBV infection. J Viral Hepat 2020;27:1096-107.

20 Eberhard JM, Kummer S, Hartjen P, et al. Reduced CD161+ MAIT cell frequencies in HCV and HIV/HCV co-infection: Is the liver the heart of the matter? J Hepatol 2016;65:1261-3.

21 Toubal A, Nel I, Lotersztajn S, et al. Mucosal-associated invariant T cells and disease. Nat Rev Immunol 2019;19:643-57.

22 Yong YK, Saeidi A, Tan HY, et al. Hyper-expression of PD-1 is associated with the levels of exhausted and dysfunctional phenotypes of circulating CD $161^{++} \mathrm{TCR}$ iV $\alpha 7.2^{+}$mucosal-associated invariant T cells in chronic hepatitis B virus infection. Front Immunol 2018;9:472.

23 Yamamoto T, Price DA, Casazza JP, et al. Surface expression patterns of negative regulatory molecules identify determinants of virus-specific CD8+ T-cell exhaustion in HIV infection. Blood 2011:117:4805-15.

24 Juno JA, Waruk JLM, Wragg KM, et al. Mucosal-associated invariant $\mathrm{T}$ cells are depleted and exhibit altered chemokine receptor expression and elevated granulocyte macrophage-colony stimulating factor production during end-stage renal disease. Front Immunol 2018:9:1076.

25 Kaufmann DE, Kavanagh DG, Pereyra F, et al. Upregulation of CTLA-4 by HIV-specific CD4p T cells correlates with disease progression and defines a reversible immune dysfunction. Nat Immunol 2007;8:1246-54.

26 Raziorrouh B, Heeg M, Kurktschiev P, et al. Inhibitory phenotype of HBV-specific CD4+ T-cells is characterized by high PD-1 expression but absent coregulation of multiple inhibitory molecules. PLoS One 2014;9:e105703.

27 Aggarwal BB, Kunnumakkara AB, Harikumar KB, et al. Signal transducer and activator of transcription-3, inflammation, and cancer: how intimate is the relationship? Ann N Y Acad Sci 2009;1171:59-76.

28 Schmidt-Arras D, Rose-John S. IL-6 pathway in the liver: from physiopathology to therapy. J Hepatol 2016;64:1403-15.

29 Wang J, Sanmamed MF, Datar I, et al. Fibrinogen-like Protein 1 is a major immune inhibitory ligand of LAG-3. Cell 2019;176:334-47.

30 Acharya N, Sabatos-Peyton C, Anderson AC. Tim-3 finds its place in the cancer immunotherapy landscape. J Immunother Cancer 2020;8:e000911. 